\title{
Virtual laboratories in optics: applications to a course on lasers
}

\section{Lionel Bertrand, Sylvain Lefebvre, Stéphane Proulx}

Lionel Bertrand, Sylvain Lefebvre, Stéphane Proulx, "Virtual laboratories in optics: applications to a course on lasers," Proc. SPIE 9664, Ninth International Topical Meeting on Education and Training in Optics and Photonics, 96641E (24 October 2005); doi: 10.1117/12.2207526

SDIE Event: Ninth International Topical Meeting on Education and Training in Optics and Photonics, 2005, Marseille, France 


\title{
Ref ETOP001
}

\section{Virtual laboratories in Optics : Applications to a course on lasers}

\author{
Lionel Bertrand, Sylvain Lefebvre and Stéphane Proulx ${ }^{1}$ \\ ${ }^{1}$ École Polytechnique de Montréal, Montréal, Canada \\ Media42, Montréal.
}

\begin{abstract}
Virtual laboratories are used to help teaching a course on lasers. The spectral emission of a laser, the Zeeman effect on the laser frequencies, the frequency stabilisation of a laser and the Lamb dip are the subjects chosen to virtually illustrate the properties of a laser. All the laboratories are very close to reality, including possible experimental missteps. The student is under the conditions he can find in the real laboratory and can spend as long as he wishes, at any time. The teacher can track the student via a data base that includes the eventual missteps which the student would have committed.
\end{abstract}

\section{Keywords}

Virtual laboratories ; simulation of a laser ; close to the reality ; simulated experimental missteps ; student tracking.

\section{Summary}

The École Polytechnique de Montréal being an engineering school, the laboratories take an important part in engineer's training and particularly understanding all the phenomena involved in the operation of a laser is made easier by the use of laboratories. However a good laboratory, modern and complete, is expensive. Moreover, in the case of a laboratory concerning lasers, it is important that the student has, at the time of making the laboratory, all the piece of information and the understanding necessary for a good learning. It follows from this that we frequently do not have a sufficient number of set-ups to satisfy at the students at the good time.

One can easily imagine that the creation of virtual laboratories could solve this problem : they can be as numerous as there are computer stations, they can be carried out anywhere and at any moment, they may be amortized quickly, and, the last but not least, they are unbreakable. However, nothing can replace the reality of a true laboratory : giving the hands-on experience, facing experimental missteps, and so on. In developing virtual laboratories, we must thus try to simulate reality as much as possible.

This is what we have done with the virtual laboratories used in the course "Introduction to lasers" of the École Polytechnique de Montréal. We have simulated real equipment which exists at the École Polytechnique, with all the actual conditions of operation. For all of them, the interface is as close as possible to reality : all appear as they really are, their operation and connections between them are simulated and many common experimental missteps are possible. The student experiments conditions of the real experiment, except for the fact that he cannot break the apparatus or operate it under extreme conditions. 
The virtual laboratories are based on the same interface but many experiments can be simulated. Here for example we give some of them.

1) Spectral emission of a laser.

First the student has to understand the meaning of the use of the different optical element needed to look at the spectral emission of a laser (polarizer, spectrum analyser, beam splitter, diode, etc). He has to make the correct BNC connections between them and to place some of them at the good place. Any error is indicated and the student has to correct it before continuing. Note that these errors, and the following, are recorded in a file only accessible to the professor.

Illustrating the cavity modes frequencies of a laser, the gain and the threshold level and the mode's polarization, this laboratory permits the student to understand how a laser emits light. The effects on the mode frequencies of the temperature variations and of a piezoelectric crystal voltage applied to the cavity, are simulated with possible experimental missteps like using wrong apparatus, displacing them to a wrong place, not respecting the necessary experimental conditions or boosting the voltage applied to the piezoelectric crystal.

The student can print his experimental results obtained from a chart recorder, analyses it and fills up a report, answering some questions and measuring some experimental parameters like as the value of the frequency threshold level, the actual mode polarization and the laser total or mode intensity.

\section{2) Zeeman effect and lasers.}

Applying a magnetic field on the laser tube, the student look at the effects on the mode frequencies : mode splitting, frequency beating and polarization changes. In this laboratory, the student must measure values and plot the curves of the variations of the Zeeman beat frequency with the intensity of the magnetic field or with the mode frequency position in the laser gain curve. The weak, but not negligible, experimental fluctuations are simulated, and the values of the experimental data change weakly and randomly from one student to another. Again the student fills up a report and evaluates the Bohr magneton.

3) Frequency stabilizations of a laser and Lamb dip measurement .

The Zeeman effect and the intensity mode variations are used to stabilize the frequency of a laser. The student activates a feedback loop acting on the piezoelectric crystal to lock its voltage to a bias value corresponding to the frequency choose to stabilize the laser. These value, for each of the way of stabilization (Zeeman beat frequency or intensity mode variations) is critical : it depends strongly on the slope of the discriminating signal and the student can see how this dependence acts on the stabilization of the laser. The student measures the frequency fluctuations of the laser mode with or without the feedback loop. He compares the two methods and decides which is better, taking into account the experimental errors associated with each.

Having stabilized the laser, the student can observe the Lamb dip by drawing the intensity of the mode as a function of the mode position in the gain curve and he deduces its value from his measurements, comparing it with the theoretical value.

These is some examples of the virtual laboratories that we can produces from our multiplatform interface. It is easy to imagine other possibilities. One important point is that all the laboratories are very close to the reality : they can be used as well as tool of practice as tool of evaluation. The second point is that many experimental missteps possibly made by a student are simulated and recorded : not only the student can learn 
of his errors but the professor can also follow the progress of the student, understand his way of reasoning and so better help it to learn.

We have tested these laboratories with some students who also used the real version. All showed an unquestionable enthusiasm for the virtual version : realism, interest, greater availability and specially the resemblance with the real set-up which makes these virtual laboratories very attractive. These virtual laboratories were also used with two classes without any difficulty. 